\title{
A comparative study of clinical management strategies for vaginal discharge in family planning and genitourinary medicine settings
}

\author{
Catriona Melville, Rak Nandwani, Alison Bigrigg, Alex D McMahon
}

Abstract

Objective To compare strategies for management of women with vaginal discharge in genitourinary medicine (GUM) and family planning (FP) settings.

Methods The setting was a centre housing both FP and GUM departments within a primary care trust in Scotland. The study participants were 200 women presenting with vaginal discharge. A randomised, controlled, crossover design was employed. Strategies typical of FP and GUM were performed on every participant in a randomised sequence. Day 1 diagnoses were made by the FP strategy (history and examination) and the GUM strategy (nearpatient microscopy added). Day 7 results were obtained from final analysis of all specimens. Days 1 and 7 results were compared with the reference standard provided by all the test results. The main outcome measures were incorrect diagnoses on Days 1 and 7.

Results On Day 1 the FP strategy resulted in significantly more incorrect diagnoses than the GUM strategy when compared with the reference standard (73 vs $32 ; p<0.001$ ). On Day 7 the GUM strategy resulted in significantly more incorrect diagnoses than the FP strategy when compared with the reference standard (32 vs $17 ; p=0.019$ ).

Conclusions Vaginal discharge can be managed effectively in community settings such as FP and primary care. The addition of near-patient microscopy produces a more accurate immediate diagnosis. The addition of a high vaginal swab for culture produces a more accurate final diagnosis. The costs of on-site microscopy must be considered.

J Fam Plann Reprod Health Care 2005; 31(1): 26-30 (Accepted 23 July 2004)

\section{Key message points}

- Vaginal discharge can be managed effectively in community settings such as family planning and primary care, in areas of low gonorrhoea prevalence, without fear of suboptimal treatment.

- Near-patient microscopy is significantly more accurate than best clinical diagnosis.

- Sending a high vaginal swab for culture improves diagnostic accuracy compared with microscopy alone.

The Sandyford Initiative, Glasgow, UK

Catriona Melville, MRCOG, DFFP, Specialist Registrar in Obstetrics and Gynaecology, West of Scotland Region

Rak Nandwani, FRCP. DFFP, Associate Director and Consultant in HIV/Genitourinary Medicine

Alison Bigrigg, PFFR, FRCS, Director and Consultant in Community Gynaecology

Robertson Centre for Biostatistics, University of Glasgow, Glasgow, UK

Alex D McMahon, PhD, Senior Statistician

Correspondence to: Dr Catriona Melville, The Sandyford Initiative, 2-6 Sandyford Place, Sauchiehall Street, Glasgow G3 7NB, UK. Tel: +44 (0) 1412118130 . Fax: +44 (0) 141211 8149. E-mail: catrionamelville@tiscali.co.uk

\section{Introduction}

Many women experience abnormal vaginal discharge at some time in their lives. ${ }^{1}$ At least $25 \%$ of women attending genitourinary medicine (GUM) clinics receive treatment for one of the three common causes of abnormal vaginal discharge: bacterial vaginosis, candidiasis and trichomoniasis. ${ }^{1}$ As an alternative to GUM services, women with vaginal discharge may choose to attend their general practitioner or a family planning (FP) or gynaecology clinic. This may become an increasingly common choice if service provision is to develop in line with the recommendations made by the English National Strategy for Sexual Health and HIV ${ }^{2}$ and the Scottish sexual health and relationships strategy. ${ }^{3}$

The management of women presenting with vaginal discharge varies according to the service and also within many services. There are two main strategies in current use in the UK. The strategy traditionally utilised in community settings such as FP and primary care, as well as in hospital settings such as gynaecology, is that of 'triple swabs' (Figure 1). These specimens are sent to the local laboratory for analysis. Using this strategy the clinician will make a presumptive initial diagnosis based on the clinical signs and symptoms and will manage the patient accordingly whilst awaiting the laboratory results. In contrast, GUM services utilise on-site laboratory facilities for near-patient microscopy of genital specimens (Figure 1). This provides an immediate microscopy-based diagnosis. Although the role of near-patient microscopy has been evaluated within the GUM setting in asymptomatic women, ${ }^{4}$ there are no studies to date that directly compare the accuracy of these different strategies in either asymptomatic or symptomatic individuals. No evidence base currently exists to confirm that on-site microscopy should be enlisted in the management of all women presenting with vaginal discharge.

Increasingly, FP and GUM services are being housed under one roof. ${ }^{5-9}$ This has highlighted the difference in approaches to the management of vaginal discharge. In order to provide an integrated service, we undertook this prospective study with the aim of introducing common evidence-based departmental protocols that could be used in our centre and in other departments providing this service.

The study was designed to assess which strategy performed better in terms of diagnostic accuracy. First, we assessed how the strategy of initial diagnosis based on clinical symptoms and signs (FP strategy) performed against the microscopy result from the GUM strategy (comparison of Day 1 diagnoses). Second, we compared the final laboratory results from the two strategies (Day 7 diagnoses).

\section{Methods}

Participants

Two hundred women attending The Sandyford Initiative GUM and FP clinics with the primary complaint of vaginal discharge were invited to participate in this study. One hundred participants were recruited from each clinic 


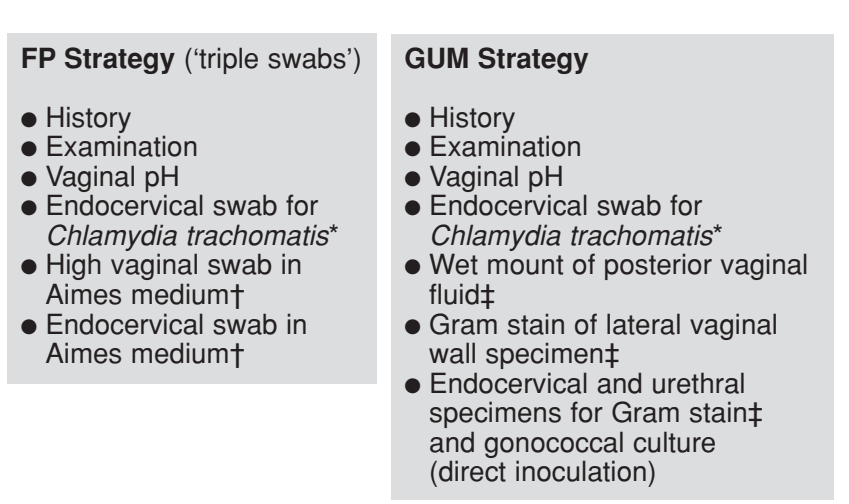

Figure 1 Strategies for the management of vaginal discharge. ${ }^{*}$ In this study both strategies used ligase chain reaction for the diagnosis of Chlamydia trachomatis. †These specimens are transported to the local laboratory for (1) culture on selective and non-selective gonococcal agar (incubation for 48 h at $37^{\circ} \mathrm{C}$ in $5 \%$ CO $)$, (2) culture in chloramphenicolincubation in Feinberg's medium for $48 \mathrm{~h}$ at $37^{\circ} \mathrm{C}$ and inverted light microscopy (for diagnosis of Trichomonas vaginalis) and (4) Gram film and microscopy. $¥$ These specimens are examined using on-site microscopy. The inoculated gonococcal plates are then transported to the local laboratory for incubation and examination. Diagnosis of $\mathrm{T}$. vaginalis and Candida is by microscopy only. Both strategies use Hay's criteria for diagnosis of bacterial vaginosis in the authors' depart FP, family planning; GUM, genitourinary medicine

between December 2001 and June 2002. Suitable participants were identified at the clinic reception desk using a tick sheet, which was used to ascertain whether the client was attending with the main complaint of vaginal discharge. Exclusion criteria comprised: antibiotic therapy within the preceding 4 weeks, pregnancy, systemic illness, genital ulceration, and known contacts of sexually transmitted infections (STIs). Inclusion criteria were: age greater than 16 years, a willingness to participate and a main complaint of vaginal discharge. No clients who were invited to participate in the study declined.

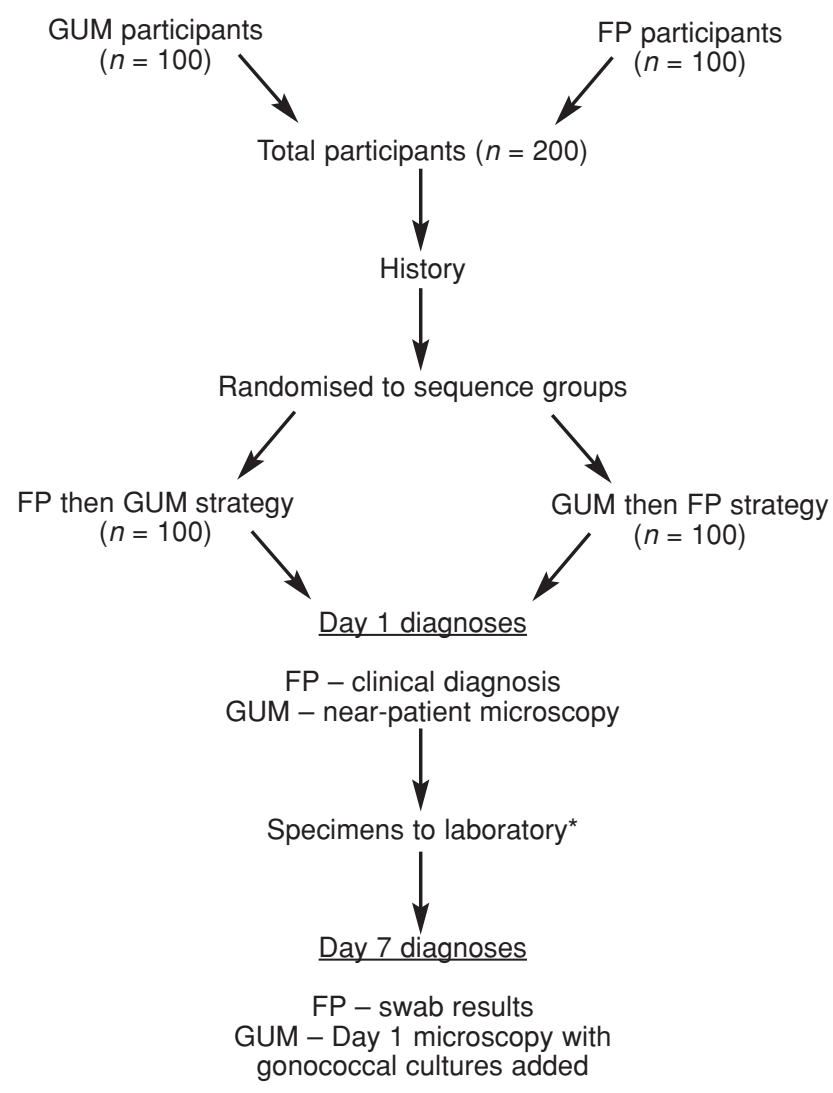

Figure 2 Study protocol. *FP swabs, GUM gonococcal plates, chlamydia specimen. GUM, genitourinary medicine; FP, family planning

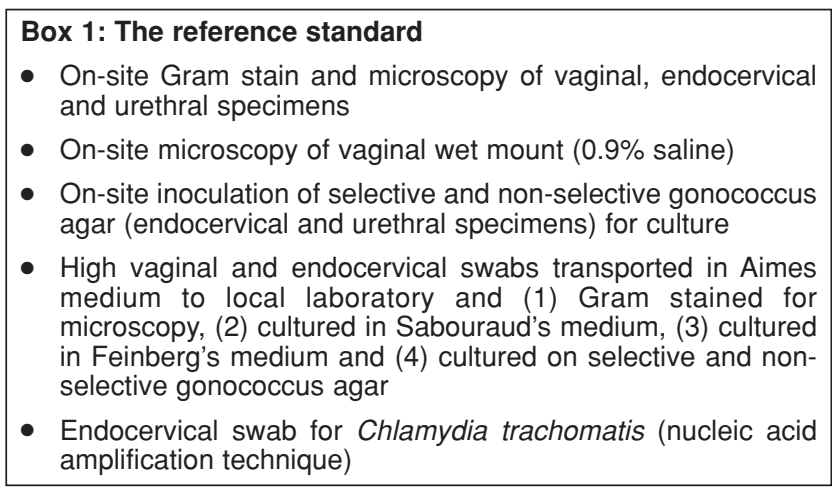

Study design

A randomised, controlled, crossover design was used. The FP and GUM strategies were performed on all participants (Figures 1 and 2). A history was taken using a standard proforma. A single researcher (C.M.) performed all the consultations and examinations. The sequence in which the strategies were performed was determined using randomly allocated numbers within independently sealed envelopes. Equal numbers of participants were randomly allocated to each sequence group (Figure 2). The researcher was blinded to the microscopy-based diagnosis until the Day 1 diagnosis from the FP strategy had been documented. To prevent suboptimal treatment, Day 1 management of each participant was based on the microscopy findings (Day 1 GUM strategy diagnosis). On Day 7 we contacted the participants with their final results and offered further treatment, if required. To determine strategy accuracy, Days 1 and 7 diagnoses were compared with the reference standard of both strategies combined (Box 1).

This study received approval from the Greater Glasgow Primary Care Trust Research Ethics Committee.

\section{Statistical analysis}

The study power was set at $80 \%$ to detect a $10 \%$ difference in strategy veracity rates. Strategy accuracy was estimated by the difference of two paired proportions, together with 95\% confidence intervals. Statistical significance was assessed by McNemar's test. If a strategy obtained only one of the diagnoses in a participant who had multiple diagnoses, it was then deemed incorrect.

Statistical comparison of FP and GUM participants and the randomised sequence groups was made using Chisquare tests and Wilcoxon tests.

\section{Results}

All participants completed the study. Table 1 summarises the characteristics of the study participants. The populations attending both clinics were similar in terms of age, ethnicity and history of an STI, but the GUM participants were significantly more likely to use no form of contraception and to smoke, and had significantly more sexual partners in the last year than did FP clinic attendees. Statistical comparison of the two randomised sequence groups was performed and no significant differences in the characteristics of these participants were found.

Table 2 summarises the number of correct and incorrect diagnoses made by each strategy on Days 1 and 7. On Day 1, the FP strategy produced 73 incorrect diagnoses and the GUM strategy produced 32 incorrect diagnoses. Statistical comparison showed that the FP strategy produced significantly more incorrect diagnoses than the GUM strategy ( $p<0.001$, difference $-20 \%$; $95 \%$ CI -27 to -14 ). On Day 7, the FP strategy produced 17 incorrect diagnoses and the GUM strategy produced 32 incorrect diagnoses. Statistical comparison showed that the GUM strategy 
ARTICLE

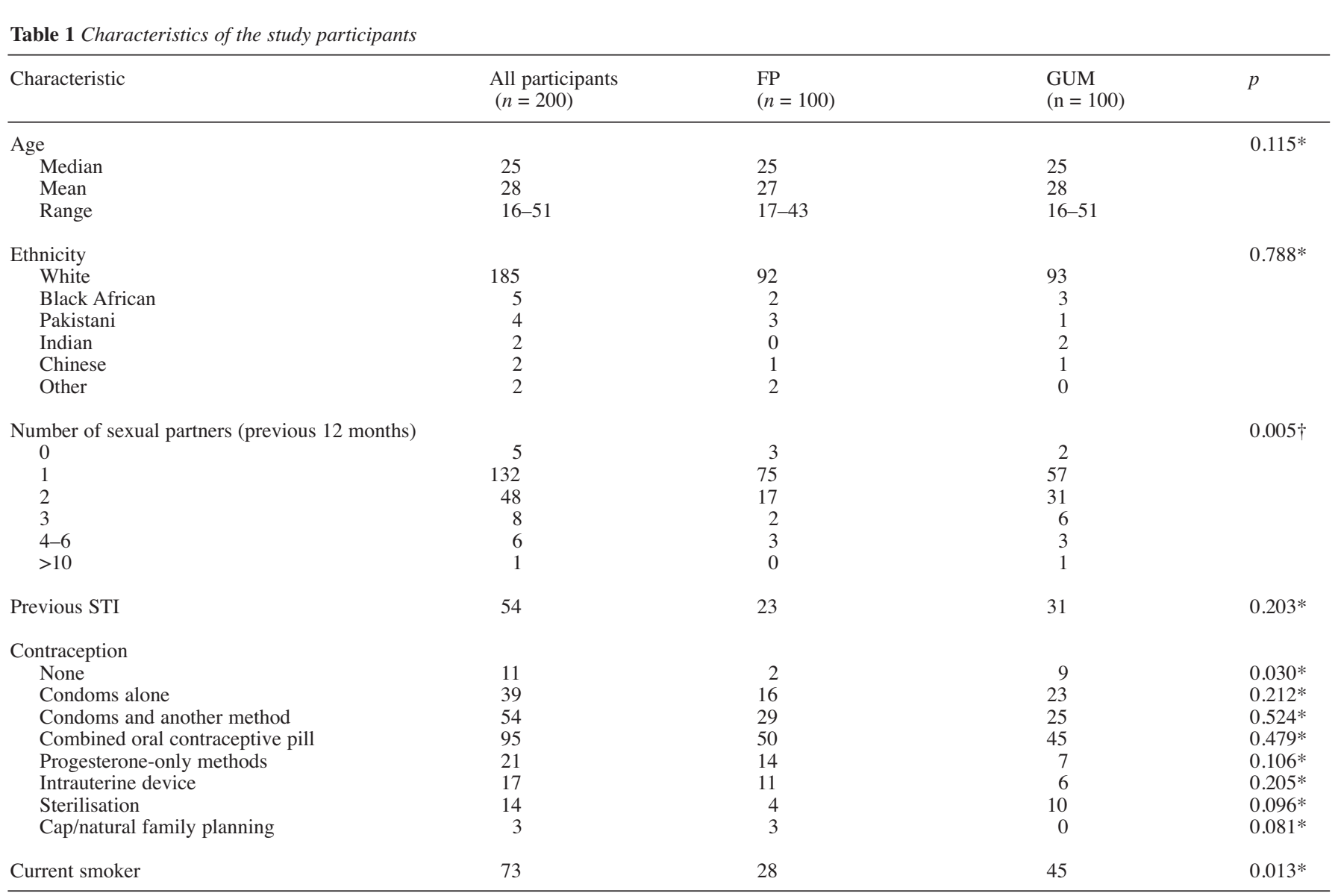

*Chi-square test. The $p$ value shows the significant difference between participants from each clinic. $†$ Wilcoxon test.

FP, participants presenting to family planning clinic; GUM, participants presenting to genitourinary medicine clinic; STI, sexually transmitted infection.

produced significantly more incorrect diagnoses than the FP strategy ( $p=0.019$, difference $7.5 \%$; 95\% CI 1.3 to 13.7 ).

As Neisseria gonorrhoeae, Chlamydia trachomatis and Group B streptococci are not invariably associated with vaginal discharge, a further analysis of the data was performed with the exclusion of these diagnoses. On Day 1, the FP strategy still had significantly more incorrect diagnoses than the GUM strategy (60 FP, 24 GUM; $p<0.001)$. On Day 7, the GUM strategy still had more incorrect diagnoses than the FP strategy (19 GUM, 12 FP). Although the trend was in the same direction as with the complete data analysis, there was less of a difference, which was no longer statistically significant $(p=0.194)$.

In order to determine if the sequence of sampling influenced diagnostic accuracy, statistical comparison of the randomised sequence groups was performed. No statistically significant difference in diagnostic accuracy was found on Days 1 or 7 ( $p=1.000$ and 0.236 , respectively).

Table 3 illustrates the specific diagnoses that were made in this study. There was no significant difference in the numbers of each infection diagnosed from each clinic. A total of 98 participants had a single diagnosis, 21 participants had two diagnoses and six participants had three or more infections diagnosed. The low prevalence of gonorrhoea in the study population is noted. This reflects the prevalence of gonorrhoea in our region.

On Day 1, the FP strategy 'over diagnosed' 27 cases of Candida albicans, 14 cases of bacterial vaginosis and one case of Trichomonas vaginalis. These infections were not shown to be present on Day 7.

The GUM strategy failed to diagnose 21 cases of $C$. albicans, 11 of streptococcus and one case of bacterial vaginosis. Thirteen of the cases of candida, five of the cases of streptococcus and the case of bacterial vaginosis were treated at Day 7 follow-up.

\section{Discussion}

Our study found that the strategy of on-site microscopy (GUM) was superior to the strategy of best clinical diagnosis (FP) in terms of initial diagnostic accuracy. The FP strategy, however, was found to be significantly more accurate than the GUM strategy in terms of final diagnosis. This is because the vaginal specimen from the GUM strategy was only examined by microscopy in-

Table 2 Correct and incorrect diagnoses made by each strategy

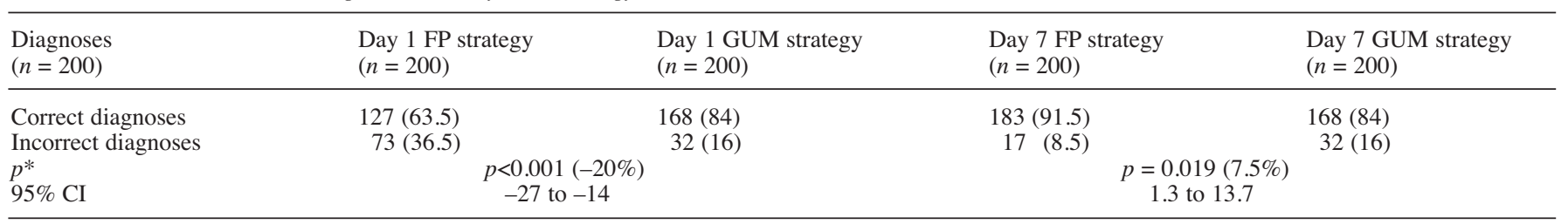

*McNemar's test. Percentage figures are given in parentheses.

$\mathrm{CI}$, confidence interval; FP, family planning; GUM, genitourinary medicine. 
Table 3 Specific diagnoses made in all participants, family planning and genitourinary medicine participants and randomised sequence groups

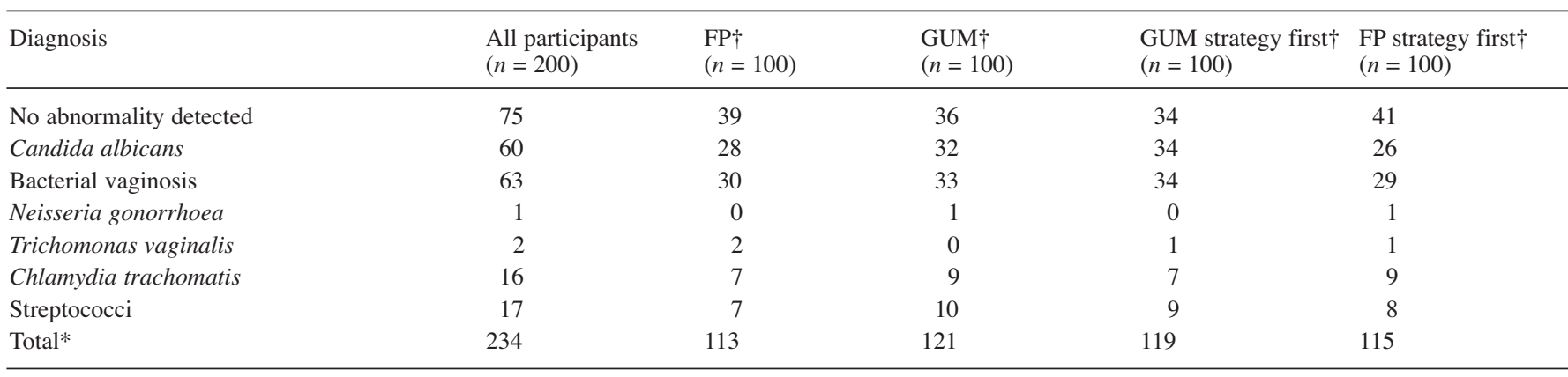

*Twenty-seven participants had multiple diagnoses, hence total number of infections $>200$. $\dagger$ Statistical comparison of the diagnoses made in participants from each clinic and in each randomised sequence group showed no significant difference (Chi-square test).

FP, family planning; GUM, genitourinary medicine.

house and not routinely plated onto culture media to yield growths of Candida and Streptococcus species. Although practices such as this are widely used throughout the UK in GUM departments, changes are being stimulated by the modernisation of services. ${ }^{10}$ We found that although the GUM and FP populations share many similar characteristics, there are some differences, which may be important. There were, however, no significant differences in the infections diagnosed in participants from each clinic.

\section{Strengths and weaknesses}

The diagnostic accuracy of these strategies had not been previously compared. For this reason we thought it unethical for a GUM participant to be denied near-patient microscopy. We therefore employed a crossover design in which all participants underwent both strategies thus minimising the chance of suboptimal treatment.

In order to eliminate sampling bias we randomised the sequence of strategies. The primary researcher performed all examinations thus avoiding observer variability. The researcher was blinded to the microscopy result until a clinical diagnosis was documented. All microscopy procedures were performed by the duty medical laboratory scientific officer. All specimens were analysed in the same laboratory and were not labelled as study specimens to reduce bias further.

In order to extrapolate our findings to routine clinical care we must assume that the researcher's clinical acumen was representative of FP staff. To confirm this, the knowledge of a sample of FP staff was assessed, and found to be comparable with the researcher's knowledge. ${ }^{11}$

To evaluate accuracy, the outcome of a test or strategy is compared with an independent reference standard. 'Gold standards' providing full certainty are rare and sometimes no suitable independent standard is available. ${ }^{12}$ No independent reference standard existed for the present study and therefore we utilised a combination of both strategies.

\section{Implications for clinical practice}

Our research was designed to compare strategy accuracy in terms of diagnosis. No comment can therefore be made regarding the clinical implication of the diagnosed infections. This is of particular relevance for the streptococcal and candidal diagnoses. Group B streptococci are known to colonise the genital tract in up to $18 \%$ of healthy women. ${ }^{13}$ Mean vaginal isolation rates for C. albicans from asymptomatic women have been reported as being 8\%.14 All participants in our study were symptomatic at recruitment, and were only offered treatment for these infections on Day 7 if still symptomatic. In order to investigate causality, further definitive studies are required, with participants being randomised to treatment or no treatment of these infections with posttreatment follow-up.

This study has shown that in terms of immediate diagnosis, microscopy is more accurate than best clinical judgement. Managing patients solely on the basis of clinical diagnosis may result in over- or incorrect treatment. Clinicians who do not have access to nearpatient testing may be reassured that the FP strategy did not miss the cases of $T$. vaginalis or $N$. gonorrhoeae. This strategy does, however, incur a delay of approximately 7 days in obtaining results. This may be acceptable in regions with low female gonorrhoea prevalence, such as ours, and is similar to the time period taken to obtain a chlamydia result. In areas of higher prevalence, however, near-patient testing may be important to limit transmission of the infection. Additionally, when questioned, the women participating in the study expressed a strong preference for microscopy results to be available on Day 1.15 This does, however, need to be balanced against the feasibility and cost of providing near-patient microscopy in non-GUM settings.

The vaginal specimens from the GUM strategy were examined by microscopy in-house but not routinely plated onto culture media. There is known to be considerable variation in many aspects of UK GUM clinical practice ${ }^{16}$ Routine high vaginal swab to augment microscopy is one such example. To increase the accuracy of this strategy, a vaginal specimen could be sent to the laboratory for culture. FP clinical practice also shows much variation, and vaginal $\mathrm{pH}$ measurement may not be routinely utilised. This procedure could be easily introduced however.

We have found that FP and GUM attendees have many similar characteristics and show no significant difference in the infections diagnosed. We therefore feel it is possible to develop a unified protocol for the management of women presenting with vaginal discharge. The conclusions of this study may serve to stimulate a review of protocols in other settings. The findings of this study have already been used to alter the authors' own clinical strategies. A vaginal specimen for culture is now taken from all symptomatic GUM attendees at our centre, and we are assessing ways to select those patients who would benefit from the addition of near-patient microscopy.

Acknowledgement

The authors would like to thank Mark Mason who performed the nearpatient microscopy.

Statements on funding and competing interests

Funding. This study was financially supported by Greater Glasgow Health Board endowment funds.

Competing interests. None identified. 
References

Hay PE. The diagnosis and management of vaginal discharge. In: Barton SE, Hay PE (eds), Handbook of Genitourinary Medicine. London, UK: Arnold, 1999; 83-95.

2 Department of Health. The National Strategy for Sexual Health and HIV. London, UK: Department of Health, 2001.

3 Scottish Executive. Enhancing Sexual Wellbeing in Scotland: A Sexual Health and Relationships Strategy. Edinburgh, UK: Scottish Executive, 2003 .

4 Andrews H, Acheson N, Huengsberg M, Radcliffe KW. The role of microscopy in the diagnosis of sexually transmitted infections in women. Genitourin Med 1994; 70: 118-120.

5 Stedman Y, Elstein M. Rethinking sexual health clinics. BMJ 1995 310: 342-343.

6 Laughlin S, Nandwani R, Ilett R, Bigrigg A. The Sandyford Initiative: creating added value to health and health care. Health Bull 2001; 59: $238-243$.

7 Wilkinson C, Hampton N, Bradbeer C. The integration of family planning and genitourinary medicine services. Br J Fam Plann 2000; 26: $187-188$

8 Dawson SGM, Callander NM, Roche CB, Kingsland TR, Desmond NM. Integrated sexual healthcare: the development and review of one model of service delivery. Int J STD AIDS 2000; 11: 428-434.

9 Jones M. Integrating family planning with genitourinary medicine: developing an holistic sexual health clinic in Eastbourne. Br J Fam Plann 2000; 26: 221-224.

10 Robinson J, Rogstad K. Modernisation of GUM/HIV services: what does it mean? Int J STD AIDS 2003; 14: 89-98.

11 Melville C, Nandwani R, Bigrigg A. Impact of the Sexually Transmitted Infections Foundation Course on the knowledge of family planning nurses and doctors (Letter). Sex Transm Infect 2003 ; family plann 346 .

12 Knottnerus JA, Weel V, Muris JWM. Evaluation of diagnostic procedures. BMJ 2002; 324: 477-480.

13 Rowen D. Streptococci and the genital tract. Int J STD AIDS 1993; 4: 63-66.

14 Sonnex C, Lefort W. Microscopic features of vaginal candidiasis and their relation to symptomatology. Sex Transm Infect 1999; 75: 417-419.

15 Melville C, Bigrigg A, Nandwani R. Client perspectives on sexual health service provision. Int J STD AIDS 2004; 15: 380-383.

16 Carne CA, Foley D, Kell P, Maw R. Variation in clinical practice in genitourinary medicine clinics in the United Kingdom. Sex Transm Infect 2003; 79: 240-242.

\section{DR KATHARINA DALTON MEMORIAL SERVICE}

A celebration of the life and works of Dr Katharina (Kittie) Dalton will take place at Rosslyn Hill Unitarian Chapel, Willoughby Road, Hampstead, London NW3 1SB on Saturday 22nd January 2005 at 2.00 pm.

All are welcome to share in the celebration and join Katharina's family and friends for refreshments afterwards. If you wish a particular item to be included in the service please contact Katharina's daughter, Wendy Holton. Tel: 01432 760993.E-mail: Holton@morlugg.freeserve.co.uk or m.dalton@btinternet.com.

\section{But is she positive?}

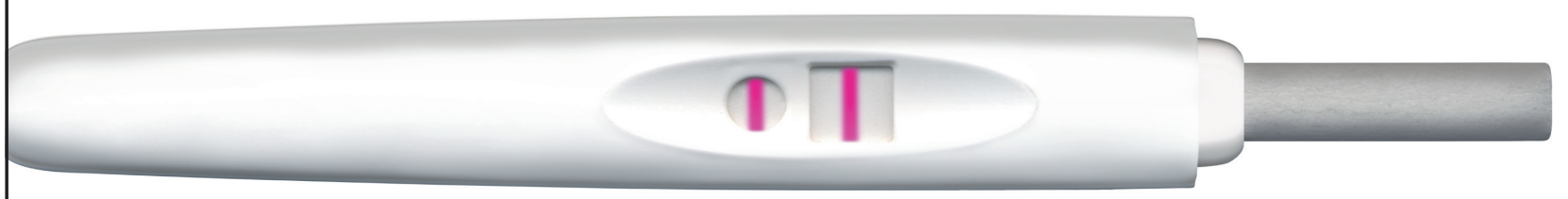

Your next patient is pregnant but doesn't want to be.

Where next? As a registered charity since 1968, bpas has offered affordable abortion care for women. We provide almost 50,000 abortions a year (including service agreements) and can offer all the professional help your patient needs.

bpas has a nationwide network of clinics and consultation centres. There are no long waits for appointments. We can offer a choice of times, clinics and procedures. All it takes to arrange an appointment is one call to the bpas Actionline on 08457304030.

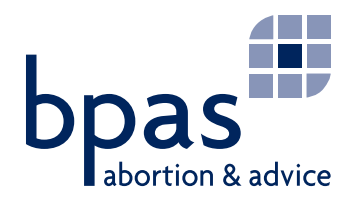

ACTIONLINE 08457304030 bpas positively the best service 